\title{
Converting S-limonene Synthase to Pinene and Phellandrene Synthases Reveals the Plasticity of the Active Site
}

\author{
Jinkun $\mathrm{Xu}^{\mathrm{a}}$, Ying $\mathrm{Ai}^{1}$, Jianhui Wang ${ }^{1}$, Jingwei $\mathrm{Xu}^{1}$, Yongkang Zhang ${ }^{1}$ \\ and Dong Yang ${ }^{1 *}$
}

${ }^{1}$ Gene Engineering and Biotechnology Beijing Key Laboratory, College of Life Sciences, Beijing Normal University, Beijing, 100875 China

*Corresponding author: yangdong@,bnu.edu.cn （ph. +86-010-58808197; fax $+86-010-58807365)$ 


\section{ABSTRACT}

S-limonene synthase is a model monoterpene synthase that cyclizes geranyl pyrophosphate (GPP) to form S-limonene. It is a relatively specific enzyme as the majority of its products are composed of limonene. In this study, we converted it to pinene and phellandrene synthases after introducing N345A/L423A/S454A or N345I mutations. Further studies on N345 suggest the polarity of this residue plays a critical role in limonene production by stabilizing the terpinyl cation intermediate. If it is mutated to a non-polar residue, further cyclization or hydride shifts occurs so the carbocation migrates towards the pyrophosphate, leading to the production of pinene or phellandrene. On the other hand, mutant enzymes that still possess a polar residue at this position produce limonene as the major product. N345 is not the only polar residue that may stabilize the terpinyl cation because it is not strictly conserved among limonene synthases across species and there are also several other polar residues in this area. These residues could form a "polar pocket" that may collectively play this stabilizing role. Our study provides important insights into the catalytic mechanism of limonene synthases. Furthermore, it also has wider implications on the evolution of terpene synthases. 
Key words

Limonene; Pinene; Phellandrene; Terpene synthase; Evolution;

Site-directed mutagenesis; Terpene diversity 


\section{INTRODUCTION}

Terpenes form one of the largest groups of natural products (Wise and Croteau, 1999; Toll, 2006; Christianson, 2006; Christianson, 2007; Degenhardt et al., 2009; Gao et al., 2012). All of them are derived from isopentenyl pyrophosphate (IPP) and its isomer dimethylallyl pyrophosphate (DMAPP). The head-to-tail condensation of DMAPP and IPP forms geranyl pyrophosphate (GPP) that can be condensed with more IPP units to form farnesyl pyrophosphate (FPP), geranylgeranyl pyrophosphate (GGPP) and longer polyprenyl diphosphates (Wise and Croteau, 1999; Toll, 2006; Christianson, 2006; Christianson, 2007; Degenhardt et al., 2009; Gao et al., 2012). These linear molecules are then cyclized by terpene synthases, creating a diverse set of carbon skeletons that are further modified to form final terpenoid products (Wise and Croteau, 1999; Toll, 2006; Christianson, 2006; Christianson, 2007; Degenhardt et al., 2009; Gao et al., 2012). Terpenes play important roles in biology, as they function as hormones, regulators of membrane fluidity (e.g., cholesterol) and insect repellants (Pare and Tumlinson, 1999; Umehara et al., 2008). They also have a variety of applications in food, medicine and chemical industries (Ajikumar et al., 2008; Kirby and Keasling, 2009).

It has been estimated that there are more than 55,000 different terpene 
metabolites (Gao et al., 2012). This striking diversity is created by terpene synthases (Wise and Croteau, 1999; Toll, 2006; Christianson, 2006; Christianson, 2007; Degenhardt et al., 2009; Gao et al., 2012). The product profile of terpene synthases is determined by the conformation of the substrate or intermediate in the active pocket (Starks et al., 1997; Christianson, 2006; Christianson, 2007; Gao et al., 2012). Stabilization of carbocations also plays a significant role (Lesburg et al., 1997; Starks et al., 1997; Christianson, 2007; Gao et al., 2012). For example, aromatic or polar side chains may stabilize the initially formed carbocation intermediate long enough for deprotonation to occur, "short-circuiting" its migration that can lead to more complicate products (Christianson, 2007; Zhou and Peters, 2011; Gao et al., 2012).

Terpenes derived from cyclization of GPP are monoterpenes. S-limonene synthase is a typical monoterpene synthase. It produces 4S-limonene, the precursor of oxygenated monoterpene in essential oils such as (-)-menthol, (-)-carvone and (-)-perillaldehyde (Guenther, 1975; Croteau et al., 2005; Hyatt et al., 2007). Previous studies have revealed its mechanism (Fig. 1; Pyun et al., 1993; Williams et al., 1998; Schwab et al., 2001; Hyatt et al., 2007). First, the pyrophosphate group migrates to C3 to form 3S-linalyl pyrophosphate (3S-LPP), which is followed by rotation about $\mathrm{C} 2-\mathrm{C} 3$ to create the cisoid conformer. This is followed by the ionization of 3S-LPP and C1-C6 cyclization, creating the 
(4S)- $\alpha$-terpinyl cation. Deprotonation of the terpinyl cation forms 4S-limonene. Although the principle product is $4 \mathrm{~S}-$ limonene $(94 \%)$, side products, including myrcene $(2 \%), \alpha$ - pinene $(2 \%)$ and $\beta$-pinene $(2 \%)$, are also produced (Croteau et al., 2005; Hyatt et al., 2007; Srividya et al., 2015). Despite the availability of its crystal structures (Hyatt et al., 2007), it is still unclear about the mechanism of its product selectivity. A previous study reported that the activity of $A$. grandis limonene synthase can be converted to that of limonene/pinene synthase by domain swapping (Katoh et al., 2004). However, this study has not identified any key residues that determine the product selectivity (Katoh et al., 2004).

In order to delineate the detailed catalytic mechanism, it is critical to identify residues that contribute to discrete steps within the reaction. One strategy is to perform systematic mutagenesis analysis (Yoshikuni et al., 2006; O'Maille et al., 2008; Srividya et al., 2015). For example, by performing a systematic alanine-scanning study on M. spicata S-limonene synthase, Srividya and colleagues have identified W423 and H579 as key residues for the stabilization and deprotonation of the terpinyl cation intermediate (Srividya et al., 2015). Other studies are focused on switching the enzyme activity by mutating only a few residues (Wilderman and Peters, 2007; Xu et al., 2007; Keeling et al., 2008; Morrone et al., 2008; Zerbe et al., 2008). Sometime this can be achieved by changing only one amino acid (Wilderman and Peters, 2007; Xu et al., 
2007; Morrone et al., 2008).

Here, we demonstrate that altering a few residues is sufficient to convert $M$. spicata S-limonene synthase to pinene synthase or phellandrene synthase. Our data further suggest that the polarity of the residue 345 and neighboring amino acids is essential for the production of limonene. Replacing them with non-polar residues may cause rearrangements of the terpinyl cation, creating more complicated products.

\section{RESULTS}

\subsection{L423A/S454A/N345A mutation creates a pinene synthase}

Some terpene synthases, such as $\gamma$-humulene synthase, are promiscuous and generate a wide range of products (Yoshikuni et al., 2006). By assuming independent and additive nature of plasticity residues, Yoshikuni and colleagues have established a design methodology that can combine several mutations to dramatically change the product selectivity of $\gamma$-humulene synthase (Yoshikuni et al., 2006).

Compared with $\gamma$-humulene synthase, S-limonene synthase is much more specific. Here we asked whether there are any plasticity residues in its active pocket. To address this question, we tried to convert this enzyme to pinene synthase by site-directed mutagenesis. An alanine-scanning mutagenesis study published by Srividya and colleagues 
has suggested that mutations N345A, L423A and S454A increase the production of pinene (Srividya et al., 2015). Based on this information and the methodology published by Yoshikuni and colleagues (Yoshikuni et al., 2006), we made two mutant proteins and named them as M2 (L423A/S454A) and M3 (N345A/L423A/S454A), respectively. GC-MS analysis indicated both mutants produce a significantly increased amount of pinene (Fig. 2a-c; Table 1). While M2 produces slightly increased amounts of pinene, M3 forms massively increased quantities of pinene at the expense of limonene. The products of M3 are composed of $21.68 \%$ $\alpha$-pinene, $47.07 \% \beta$-pinene and only $9.19 \%$ limonene. Therefore, M3 is essentially a pinene synthase as about $70 \%$ of its products are composed of pinenes.

Studies on various terpene synthases, including aristolochene synthase and taxadiene synthase, suggest that the active pocket places steric constraints on the reaction intermediate and can determine the product profile (Shishova et al., 2007; Köksal et al., 2011; Gao et al., 2012). Therefore, an enlargement of the active pocket may release this constraint and make the enzyme more promiscuous (Shishova et al., 2007; Köksal et al., 2011; Gao et al., 2012). This may explain why alanine mutations described here can facilitate the second ring closure (2, 7-ring closure) for pinene formation. 
We asked whether a further enlargement of the active pocket can lead to the formation of more pinene. Two mutants, M4 $(\mathrm{N} 345 \mathrm{G} / \mathrm{L} 423 \mathrm{~A} / \mathrm{S} 454 \mathrm{~A})$ and M5 (N345A/L423A/S454G), were generated. The products of M4 are composed of $18.81 \% \alpha$-pinene, $44.00 \%$ $\beta$-pinene, and $16.2 \%$ limonene, while the products of M5 are composed of 9.8\% $\alpha$-pinene, $52.7 \% \beta$-pinene and 13.4\% limonene (Fig. $2 \mathrm{~d}$; Table 1). Therefore, the further enlargement of the active pocket does not increase the pinene production as a percentage of total terpene products. However, compared with M3, M5 has an about two-fold increase in the yield of overall terpene products (Table 1).

\subsection{The residue at the 345 position plays a crucial role in the product}

\section{selectivity}

The difference between M2 and M3 is the mutation N345A. The fraction of pinene within terpene products increases from about $15 \%$ in M2 to nearly $70 \%$ in M3. Furthermore, in addition to pinene, products of M3, M4 and M5 contain a significant amount of $\beta$-phellandrene (Table 1). The wild type S-limonene synthase and M2, however, only make a negligible amount of this compound (Table 1). Therefore, the residue N345 seems to be important for the product selectivity of M. spicata S-limonene synthase.

To further elucidate this issue, we introduced a bulky side chain at this site by introducing the N345I mutation. Mutant N345I does not 
produce $\beta$-pinene and makes a much smaller amount of $\alpha$-pinene (Fig. 3a; Table 1). However, it produces more $\beta$-phellandrene (39.4\%) and, surprisingly, also produces $\alpha$-phellandrene (29.47\%), a terpene that is in a negligible amount in products of earlier mutants (Table 1). Indeed the major products of N345I (about 68\%) are composed of phellandrenes. Therefore, this mutant essentially becomes a phellandrene synthase.

Since both N345A (Srividya et al., 2015) and N345I cause a significant decrease in the production of limonene, we asked whether the polarity of this residue is important for limonene production. To address this question, we made N345L, N345S and N345D mutants. The two mutants that contain a polar reside at the 345 position (N345S and N345D) predominantly produce limonene, while the mutant that has a none-polar residue at this site $(\mathrm{N} 345 \mathrm{~L})$ shows a great decrease in limonene production (Fig. 3b-d; Table 1). Therefore, a polar residue in this position seems to be essential for limonene production and this effect is retained even in residues with a small side chain, such as Ser.

$2.3 N 345$ is not the sole polar residue that is essential for limonene synthesis

By sequence analysis, we observed N345 is not strictly conserved among limonene synthases (Fig. 4). In many species, this residue can be Ile or Cys instead. Therefore, it is possible that other polar residues in the active pocket may play the same role. Here, we noticed an interesting 
relationship between residues at the locations equivalent to residues 345 and 321 in the M. spicata enzyme. In enzymes with an Asn at the 345 position, the residue at the 321 position is always Cys. On the other hand, if an Ile or Cys is at the former site, the residue at the later position is always Ser or Tyr, respectively. Therefore, it seems that a more polar residue at the 321 position could compensate for the loss of polarity at the 345 position. To test this hypothesis, we created M6 by introducing a C321Y mutation in M3. Our data shows its products are composed of 40.34\% limonene, a four-fold increase from M3 (Table 1). This result indicates Y321 does provide some polar effects essential for producing limonene.

Furthermore, the crystal structure of $M$. spicata limonene synthase in complex with 2-fluoro-linalyl pyrophosphate (FLPP) (2ONH; Hyatt et al., 2007) reveals several polar residues close to the $C 7$ atom of the ligand. These include C321, W324, N345, T349, S454, M458, H579 and Y573 (Fig. 5a). These residues may collectively contribute to limonene formation. This notion is supported by the study from Srividya and colleagues that shows a significant decrease in limonene production after mutating many of these residues to Ala. For example, S-limonene only accounts for $18.1 \%, 20.9 \%, 60.3 \%, 73.7 \%, 3.2 \%$ and $56.3 \%$ of the products made by W324, N345A, T349A, S454A, M458A and H579A, respectively (Srividya et al., 2015). For further validation, we made the 
mutant S454G and found its products are composed of about $46 \% \alpha-$ and $\beta$-pinenes and only about 52\% limonene (Table 1 ). Compared with S454A, S454G produces less limonene and also has a more than two-fold increase in pinene production (Srividya et al., 2015).

\section{DISCUSSION}

Studies on bornyl pyrophosphate synthase, ent-kaurene synthase, syn-pimara-7, 15-diene synthase and abietadiene synthase suggest the co-product pyrophosphate makes strong electrostatic interactions with the carbocation intermediate and steers the migration of carbocation towards itself (Wilderman and Peters, 2007; Xu et al., 2007; Morrone et al., 2008; Zhou and Peters, 2011; Gao et al., 2012). From the crystal structure, we determined the distance between $\alpha$-phosphorus and C3, C5 and C7 to be $2.72 \AA, 4.70 \AA$ and $6.43 \AA$, respectively. Since during 2,7-ring closure and 5,7- or 1,7-hydride shift the carbocation is migrated from $\mathrm{C} 7$ to $\mathrm{C} 3$ or $\mathrm{C} 5$ (Fig. 1), the formation of pinene and phellandrene does involve the migration of carbocation towards the pyrophosphate. This suggests the pyrophosphate also plays a driving role during the synthesis of pinene and phellandrene in our mutants.

It has been established that, in certain terpene synthases, by stabilizing the initially formed carbocation a polar residue may prevent its migration towards the pyrophosphate (Wilderman and Peters, 2007; Xu et 
al., 2007; Morrone et al., 2008; Zhou and Peters, 2011; Gao et al., 2012). On the other hand, if this residue is replaced with a none-polar amino acid, this stabilization effect is lost and carbocation migration occurs, leading to the formation of more complicated products. For example, in the rice ent-kaurene synthase, by mutating an Ile to Thr one can switch its activity to that of ent-pimaradiene synthase (Xu et al., 2007).

In M. spicata limonene synthase, it seems that the polar side chain of N345 plays the same stabilizing role. Therefore, instead of migrating towards the pyrophosphate, the terpinyl cation is directly deprotonated to limonene. If N345 is replaced with Ser, because of its shorter side chain, this effect is weaker. This explains the decrease in limonene production from more than $90 \%$ to less than $70 \%$ in N345S (Fig. 3d; Table 1). Replacing N345 with none-polar residues, such as Gly, Ala, Leu and Ile, abolishes this effect and allows the migration of carbocation, which leads to the formation of more complicated products. As mentioned in 2.3, there are many polar residues in the area that may be collectively responsible for limonene formation. Here we propose that all these polar residues may form a "polar pocket" that contributes to the stabilization of the terpinyl cation. Therefore, mutating any of them to non-polar residues may disrupt the "polar pocket" and allow the migration of carbocation, which leads to the decrease of limonene production and increase of side products. Among these residues, W324 is also an aromatic residue. 
Therefore, its effect may also be caused by the aromaticity of its side chain. Furthermore, this residue might play more roles than stabilizing carbocation, as its mutation to Ala leads to a significant increase in linalool (Srividya et al., 2015). For example, W324 might be required for bringing $\mathrm{C} 1$ and $\mathrm{C} 6$ in close proximity for cyclization. W324A mutation might also cause a sufficient increase in the volume of the active site, which allows the presence of a water molecule necessary to produce linalool.

The final product profile, however, is influenced by the geometry of the active pocket. For example, steric hindrance may place a severe limitation on 2,7-ring closure. Therefore, enlarging the active pocket by N345A, S454A or S454G mutations can create more steric freedoms and significantly enhance the pinene production. This also explains why S454G produces more pinenes than S454A, because with a smaller side chain S454G possesses a larger active pocket than S454A. Although L423 is not a residue directly residing in the active pocket, as a second-tier residue it contacts S454. Therefore, its mutation to Ala may lead to an outward movement of the loop containing resides I453, S454 and G455 and enlarge the active pocket (Fig. 5b). Changing of product profile by mutating second-tier residues has been well documented by Greenhagen and colleagues (Greenhagen et al., 2006). On the other hand, hydride shifts may be less affected by steric hindrance. This explains why 
N345I produces much more phellandrene than pinene. The behavior of M6 may also be explained this way. Its products are also composed of $60 \%$ sabinene (Table 1). Probably, the bulky side chain of Y321 causes steric hindrance that prevents 2,7-ring closure but still allows 6,7-hydride shift and 2,6-ring closure.

Phylogenetic analysis on terpene synthases recognizes seven major clades, including TPS-a, b, c, d, e/f, g and h (Chen et al., 2011). The clade c is composed of copalyl synthase/kaurine synthase (CPS/KS) in $P$. patens as well as CPS from both gymnosperms and angiosperms. This clade is supposed to be the base of the tree. The clade b contains cyclic monoterpene synthases from angiosperms, while the clade $d$ comprises gymnospermic mono-, sesqui- and di-terpene synthases. Therefore, it seems that monoterpene synthases from angiosperms and gymnosperms evolved independently. After we constructed a phylogenetic tree of limonene, pinene and phellandrene synthases, it is clear that the majority of limonene synthases in angiosperms form a distinct branch within the clade b (Fig. 4). The member within this branch has a more conserved "polar pocket". On the other hand, a few angiospermic limonene synthases outside this branch (e.g., from L. angustifolia and R. officinalis) as well as all gymnospermic limonene synthases belong to many branches intermingling with branches leading to pinene and phellandrene synthases and have more variations in "polar pocket" residues (Fig. 4). Therefore, 
we hypothesize that during the evolution of angiosperms, this "polar pocket" was "fine-tuned", which led to the appearance of the branch containing most angiospermic limonene synthases from ancestral limonene/pinene/phellandrene synthases. This scenario is consistent with the notion that specific activities may evolve from catalytically promiscuous ancestors (Bohlmann et al., 1998; O'Brien and Herschlag, 1999; Copley, 2003; O'Maille et al., 2008).

Our data support the previous hypothesis that new terpene synthases evolved from existing enzymes by changing a very small number of amino acids (some time a single residue) (Wilderman and Peters, 2007; Xu et al., 2007; Morrone et al., 2008; Zhou and Peters, 2011; Gao et al., 2012). If the new terpenoid produced by the mutant enzyme can confer a benefit to the plant, its gene may be selected. And during the course of evolution, both of its catalytic efficiency and product specificity will be increased as more beneficial mutations are selected. In our study, the first set of three mutations (N345A/L423A/S454A) created a pinene synthase out of limonene synthase (M3). By introducing one more mutation (S454G), we made M6 and raised the activity of the mutant enzyme to nearly $200 \%$ of that of its parent (Table 1 ). This clearly demonstrates the relative ease to evolve a new terpene sythase by mutations.

\section{CONCLUSIONS}


In conclusion, we converted the S-limonene synthase from $M$. spicata to pinene synthase or phellandrene synthase by mutating a small number of residues, confirming the plasticity of its active site. Furthermore, our data revealed that polar residues in its active pocket ensure its product selectivity for limonene by stabilizing the terpinyl cation. Our findings will have wider implications on the evolution of the chemical diversity of terpenoid products.

\section{EXPERIMENTAL PROCEDURES}

\subsection{Chemicals}

Dithiothreitol (DTT) was from Merck (Germany). Tryptone and yeast extract were from Oxoid (UK). Tris and Mopso were from AMERSCO (USA). BL21 (DE3) and the site-Directed Mutagenesis Kit were from TransGen Biotech (China). Glutathione was from J\&K Chemical (China). Glutathione Sepharose resin was from GE Healthcare Life Sciences (USA). Thrombin was from CALBIOCHEM (USA). Isopropyl $\beta$-D-thiogalactoside (IPTG), geranyl diphosphate (GPP), phenylmethylsulfonyl fluoride (PMSF), EDTA and all other chemicals were from Sigma Aldrich (USA).

\subsection{Gene synthesis and Mutagenesis}

The gene sequences encoding $M$. spicata S-limonene synthase were optimized for expression in E.coli and synthesized by Sangon Biotech 
(China). Its N-terminal transit peptide was truncated by subcloning the region encoding the amino acid sequence from $\mathrm{R} 58$ to the $\mathrm{C}$-terminus into the expression vector pGEX-4T-1 according to the study by Williams and colleagues (Williams et al., 1998). Mutations were introduced using the site-directed mutagenesis kit. PCR conditions included an initial denaturation step at $95^{\circ} \mathrm{C}$ for 2 min, followed by 18 cycles (denaturation at $95^{\circ} \mathrm{C}$ for $50 \mathrm{~s}$, annealing at $60^{\circ} \mathrm{C}$ for $50 \mathrm{~s}$ and extension at $68^{\circ} \mathrm{C}$ for $7 \mathrm{~min}$ ) and a final step at $68^{\circ} \mathrm{C}$ for $7 \mathrm{~min}$. The product was digested with DpnI at $37^{\circ} \mathrm{C}$ for 2 hours. Constructs were transformed into E.coli expression strain BL21(DE3) and correct mutants were identified by gene sequencing.

\subsection{Protein expression and purification}

For expression, cells were grown in LB media to mid-log phase and expression was induced by adding $0.5 \mathrm{mM}$ IPTG at $20^{\circ} \mathrm{C}$ for $16-20$ hours. Cells were harvested, resuspended in $25 \mathrm{mM}$ Tris ( $\mathrm{pH} 8.0$ ), $150 \mathrm{mM} \mathrm{NaCl}$, 1mM DTT, 0.2mM PMSF and lysed by sonication. Lysate was applied to glutathione Sepharose resin and eluted with $25 \mathrm{mM}$ Tris (pH 8.0), $150 \mathrm{mM}$

$\mathrm{NaCl}, 1 \mathrm{mM}$ DTT, $0.5 \mathrm{mM}$ EDTA after on-column cleavage by thrombin. Protein purity was verified by SDS-PAGE.

\subsection{The GC-MS assay}

The reaction system in a total volume of $400 \mu \mathrm{l}$ contains $25 \mathrm{mM}$ Mopso (pH 7.0), 15 mM MgCl $2,1.0 \mathrm{mM} \mathrm{DTT,} \mathrm{10 \%} \mathrm{(v/v)} \mathrm{glycerol,} 68.5$ 
$\mu \mathrm{M}$ GPP and $2 \mu \mathrm{g}$ S-limonene synthase. The reaction was performed at $37^{\circ} \mathrm{C}$ for 40 minutes. To trap the limonene, $400 \mu \mathrm{l}$ hexane was added on top of the reaction mixture and vortexed for 30 minutes. The mixture was centrifuged to separate the organic layer. $1 \mu$ of hexane containing the analytes was analyzed by GC-MS (Thermo Trace GC Ultra \& ISQ). The sample was injected into a HP-88 column $100 \mathrm{~m}$ × $250 \mu \mathrm{M} \times 0.20 \mu \mathrm{M}$ thickness (Agilent). Helium (ultra purity) at a flow rate $1.0 \mathrm{ml} / \mathrm{min}$ was used as a carrier gas. The oven temperature was first kept constant at $50^{\circ} \mathrm{C}$ for $1 \mathrm{~min}$, and then increased to $130^{\circ} \mathrm{C}$ at the increment of $3^{\circ} \mathrm{C} / \mathrm{min}$, then increased to $220^{\circ} \mathrm{C}$ at the increment of $50^{\circ} \mathrm{C} / \mathrm{min}$, and finally held at this temperature for $5 \mathrm{~min}$. The injector and transfer line temperatures were set at $250^{\circ} \mathrm{C}$. Monoterpenes were identified from mass spectra and GC retention times by comparing with available authentic standards (S-limonene, $\alpha$-pinene and $\beta$-pinene) and spectra in libraries ( $\alpha$-phellandrene, $\beta$-phellandrene and sabinene). The proportion of each product was based on the ratio of the relative peak abundance. The relative activity is calculated by dividing the total peak area for terpene products of one particular enzyme by that of the wild type limonene synthase.

\section{ACKNOWLEDGEMENTS}

This work is supported by grants from the Fok Ying Tung Education 
Foundation (\#132025), the National Science Foundation of China (\#31070682 and \#31640004), the Beijing Natural Science Foundation (\#5154029), the Fundamental Research Funds for the Central Universities (2015KJJCB19), the Scientific Research Foundation for the Returned Overseas Chinese Scholars, State Education Ministry and the Program of the Co-Construction with Beijing Municipal Commission of Education of China.

\section{FIGURE LEGENDS}

Fig. 1. Proposed reaction mechanism for the synthesis of limonene, pinene, phellandrene and sabinene. .

Fig. 2. Remodeling of plasticity residues to create a pinene synthase. Chromatograms in $\mathbf{a}, \mathbf{b}, \mathbf{c}$ and $\mathbf{d}$ show the GC-MS analysis of terpene production for wild type enzyme, M2, M3 and M5, respectively. The numbers in each peak correspond to limonene (1), $\alpha$-pinene (2), $\beta$-pinene (3), $\alpha$ - phellandrene (4) , $\beta$ - phellandrene (5) and sabinene (6).

Fig. 3. Mutation analysis suggests the polarity of the residue 345 plays a key role in limonene production. Chromatograms in $\mathbf{a}, \mathbf{b}, \mathbf{c}$ and $\mathbf{d}$ show the GC-MS analysis of terpene production for N345I, N345D, N345L and N345S, respectively. The numbers in each peak correspond to 
limonene (1), $\alpha$-pinene (2), $\beta$-pinene (3), $\alpha$ - phellandrene (4) , $\beta$ phellandrene (5) and sabinene (6).

Fig. 4. Phylogenetic tree of limonene synthases, pinene synthases and phenllandrene synthases. The tree was reconstructed using maximum likelihood phylogenetic analysis in Mega 6. Limonene, pinene and phenllandrene synthases are represented by LimS, PinS and PheS, respectively. Clades TPS-b, $-\mathrm{d}$ and $-\mathrm{e} / \mathrm{f}$ are in blue, red and grey, respectively. Numbers on each branch are the bootstrap values of that branch. Terpene synthases within the box are from gymnosperms and the rest are from angiosperms. The residue numbers on top are corresponding to those in the "polar pocket" of M. spicata limonene synthase. The gene bank accession numbers of enzymes are given in the figure and they are from the following species. AGN90902.1: Mentha arvensis; AGN90903.1: Mentha x gracilis; AGN90908.1: Mentha Canadensis; AAC37366.1: Mentha spicata; AAD50304.1: Mentha longifolia; AAG01140.2: Schizonepeta tenuifolia ; AAL17636.1: Agastache rugosa; AAF65545.1: Perilla citriodora; AAG31438.1 : Perilla frutescens; ADQ73631.1: Lavandula angustifolia; Q2XSC6.1: Lavandula angustifolia; ABP01684.1: Rosmarinus officinalis; ABD77416.a: Rosmarinus officinalis; NP_001233809.1: Solanum lycopersicum; NP_001268167.1: Vitis vinifera; AFQ23190.1: Gossypium hirsutum; ANA91931.1: Paeonia lactiflora; AAB70907.1: Abies grandis; AAK83565.1: Abies grandis; 
AFU73854.1: Pinus banksiana; AAS47694.1: Picea abies; Q9M7D1.1: Abies grandis; AAB71085.1: Abies grandis; AFU73843.1: Pinus banksiana; ADZ45506.1: Picea sitchensis; AAS47692.1: Picea abies; AAP72020.1: Picea sitchensis; ADZ45507.1: Picea glauca; AFJ67816.1: Solanum habrochaites; NP_001310383.1: Solanum pennellii

Fig. 5. Structural basis for product selectivity. a. Polar residues close to the C7 atom of FLPP are shown and colored according to atom types (green: carbon; red: oxygen; blue: nitrogen; yellow: sulfur). FLPP is also shown and its carbon atom is in cyan, phosphorus is in orange, oxygen is in red and fluorine is in light blue. b. The ribbon of M. spicata limonene synthase is in orange. The Van der Waals surfaces of N345, L423 and S454 are presented and colored in the same way as the residues (green: carbon; red: oxygen; blue: nitrogen). The FLPP is colored in the same way as in $\mathbf{a}$. 


\section{TABLES}

Table 1 Product profiles and relative activities of the wild type limonene synthase and mutant enzymes.

Each data point is the mean of three sets of independent experiments and standard deviations are given. ND, production not detected.

\begin{tabular}{ccccccc}
\hline & $\begin{array}{c}\text { RT=7.14 } \\
\boldsymbol{\alpha} \text {-Phellandrene }\end{array}$ & $\begin{array}{c}\mathbf{R T}=\mathbf{7 . 4 5} \\
\boldsymbol{\alpha} \text {-pinene }\end{array}$ & $\begin{array}{c}\mathbf{R T}=\mathbf{8 . 8 3} \\
\boldsymbol{\beta} \text {-Phellandrene }\end{array}$ & $\begin{array}{c}\mathbf{R T}=\mathbf{9 . 0 1} \\
\boldsymbol{\beta} \text {-pinene }\end{array}$ & $\begin{array}{c}\mathbf{R T}=\mathbf{9 . 4 8} \\
\text { sabinene }\end{array}$ & $\begin{array}{c}\text { RT=11.05 } \\
\text { limonene }\end{array}$ \\
\hline WT & $\mathrm{ND}$ & $1.39 \pm 0.30$ & $0.31 \pm 0.06$ & $1.09 \pm 0.18$ & $0.52 \pm 0.15$ & $96.69 \pm 0.29$ \\
M2 & $\mathrm{ND}$ & $5.02 \pm 0.95$ & $1.31 \pm 0.33$ & $10.02 \pm 1.98$ & $1.46 \pm 0.22$ & $82.19 \pm 2.36$ \\
M3 & $0.77 \pm 0.090$ & $21.68 \pm 2.64$ & $17.72 \pm 3.40$ & $47.07 \pm 3.87$ & $3.58 \pm 1.67$ & $9.19 \pm 1.57$ \\
M4 & $1.41 \pm 0.15$ & $18.81 \pm 2.19$ & $17.57 \pm 1.95$ & $44.00 \pm 3.48$ & $1.99 \pm 0.17$ & $16.22 \pm 3.66$ \\
M5 & $0.90 \pm 0.15$ & $9.84 \pm 0.52$ & $17.90 \pm 1.40$ & $52.70 \pm 2.78$ & $5.29 \pm 0.21$ & $13.37 \pm 0.95$ \\
M6 & ND & ND & ND & ND & $59.66 \pm 8.31$ & $40.34 \pm 8.31$ \\
N345I & $29.47 \pm 1.18$ & $11.79 \pm 1.32$ & $39.40 \pm 1.61$ & ND & $0.85 \pm 0.24$ & $18.48 \pm 0.81$ \\
N345D & ND & $3.55 \pm 0.33$ & $2.61 \pm 0.09$ & $2.91 \pm 0.15$ & $0.95 \pm 0.09$ & $89.97 \pm 0.47$ \\
N345L & $1.70 \pm 0.19$ & $25.08 \pm 0.81$ & $20.13 \pm 0.36$ & $26.03 \pm 1.68$ & $2.01 \pm 0.58$ & $25.04 \pm 1.76$ \\
N345S & ND & $7.12 \pm 0.23$ & $15.47 \pm 0.14$ & $6.69 \pm 0.59$ & $0.88 \pm 0.20$ & $69.84 \pm 0.73$ \\
S454G & $0.35 \pm 0.11$ & $11.88 \pm 1.26$ & $0.42 \pm 0.11$ & $34.22 \pm 5.36$ & ND & $52.13 \pm 6.57$ \\
\hline
\end{tabular}

\begin{tabular}{cc}
\hline & Relative activity (\%) \\
\hline WT & $100.00 \pm 13.46$ \\
M2 & $45.97 \pm 9.55$ \\
M3 & $17.93 \pm 0.92$ \\
M4 & $14.82 \pm 0.17$ \\
M5 & $35.71 \pm 7.77$ \\
M6 & $1.52 \pm 0.18$ \\
N345I & $23.91 \pm 2.09$ \\
N345D & $52.68 \pm 2.46$ \\
N345L & $4.91 \pm 0.12$ \\
N345S & $8.4 \pm 1.68$ \\
S454G & $107.47 \pm 3.33$ \\
\hline
\end{tabular}




\section{REFERENCES}

Ajikumar, P.K., Tyo, K., Carlsen, S., Mucha, O., Phon, T.H., Stephanopoulos, G., 2008. Terpenoids: opportunities for biosynthesis of natural product drugs using engineered microorganisms. Mol. Pharmaceutics. 5, 167-190.

Bohlmann, J., Steele, C.L., Croteau, R., 1997. Monoterpene synthases from grand fir (Abies grandis). J. Biol. Chem. 272, 21784-21792.

Bohlmann, J., Meyer-Gauen, G., Croteau, R., 1998. Plant terpenoid synthases: molecular biology and phylogenetic analysis. Proc. Natl. Acad. Sci. USA 95, 4126-4133.

Bohlmann, J., Phillips, M., Ramachandiran, V., Katoh, S., Croteau, R., 1999. cDNA cloning, characterization, and functional expression of four new monoterpene synthase members of the Tpsd gene family from Grand fir (Abies grandis). Arch. Biochem. Biophys. 368, 232-243.

Chen, F., Tholl, D., Bohlmann, J., Pichersky, E., 2011. The family of terpene synthases in plants: a mid-size family of genes for specialized metabolism that is highly diversified through the kingdom. Plant J. 66, 212-229.

Christianson, D.W., 2006. Structure biology and chemistry of the terpenoid cyclases. Chem. Rev. 106, 3412-3442. 
Christianson, D.W., 2007. Roots of biosynthetic diversity. Science 316, $60-61$.

Copley, S.D., 2003. Enzymes with extra talents: moonlighting functions and catalytic promiscuity. Curr. Opin. Chem. Biol. 7, 265-272.

Croteau, R.B., Davis, E.M., Ringer, K.L., Wildung, M.R., 2005. (-)-Menthol biosynthesis and molecular genetics. Naturwissenschaften 92, $562-577$

Degenhardt, J., Köllner, T.G., Gershenzon, J., 2009. Monoterpene and sesquiterpene synthases and the origin of terpene skeletal diversity in plants. Phytochemistry 70, 1621-1637.

Gao, Y., Honzatko, R.B., Peters, R.J., 2012. Terpenoid synthase structures: a so far incomplete view of complex catalysis. Nat. Prod. Rep. 29, $1153-1175$.

Greenhagen, B.T., O'Maille, P.E., Noel, J.P., Chappell, J., 2006. Identifying and manipulating structural determinates linking catalytic specificities in terpene synthases. Proc. Natl. Acad. Sci. USA 103, 9826-9831.

Guenther, B., 1975. In: The essential oils. RE Kreiger, Huntington, NY, Vol 2, pp. 22-27.

Hyatt, D.C., Youn, B., Zhao, Y., Santhamma, B., Coates, R.M., Croteau, R.B., Kang, C., 2007. Structure of limonene synthase, a simple model for terpenoid cyclase catalysis. Proc. Natl. Acad. Sci. USA 104, 5360-5365. 
Katoh, S., Hyatt, D., Croteau, R., 2004. Altering products outcome in Abies Grandis (-)-limonene synthase and (-)-limonene/(-)-pinene synthase by domain swapping and direct mutagenesis. Arch. Biochem. Biophys. 425, 65-76.

Keeling, C.I., Weisshaar, S., Lin, R.P.C., Bohlmann, J., 2008. Functional plasticity of paralogous diterpene synthases involved in conifer defense. Proc. Natl. Acad. Sci. USA 105, 1085-1090.

Kirby, J. and Keasling, J.D., 2009. Biosynthesis of plant isoprenoids: perspectives for microbial engineering. Annu. Rev. Plant. Biol. 60, $335-355$.

Köksal, M., Jin, Y., Coates, R.M., Croteau, R., Christianson, D.W., 2011. Taxadiene synthase structure and evolution of modular architecture in terpene biosynthesis. Nature 469, 116-120.

Lesburg, C.A., Zhai, G., Cane, D.E., Christianson, D.W., 1997. Crystal structure of pentalenene synthase: mechanistic insights on terpenoid cyclization reactions in biology. Science 277, 1820-1824.

Morrone, D., Xu, M., Fulton, D.B., Determan, M.K., Peters, R.J., 2008. Increasing complexity of a diterpene synthase reaction with a single residue switch. J. Am. Chem. Soc. 130, 5400-5401.

O'Brien, P.J., Herschlag, D., 1999. Catalytic promiscuity and the evolution of new enzymatic activities. Chem. Biol. 6, R91-R105. O’Maille, P.E., Malone, A., Dellas, N., Hess Jr, B.A., Smentek, L., 
Sheehan, I., Greenhagen, B.T., Chappell, J., Manning, G., Noel, J.P., 2008. Quantitative exploration of the catalytic landscape separating divergent plant sesquiterpene synthases. Nat Chem Biol 4, 617-623.

Pare, P.W., Tumlinson, J.H., 1999. Plant volatiles as a defence against insect herbivores. Plant. Physiol. 121, 325-331.

Pyun, H.-J., Coates, R.M., Wagschal, K.C., McGeady, P., Croteau, R.B., 1993. Regiospecificity and isotope effects associated with the methy-methylene eliminations in the enzyme-catalyzed biosynthesis of (R)- and (S)-limonene. J. Org. Chem. 58, 3998-4009.

Schwab, W., Williams, D.C., Davis, E.M., Croteau, R., 2001. Mechanism of monoterpene cyclization: stereochemical aspects of the transformation of noncyclizable substrates analog by recombinant (-)-limonene synthase, (+)-bornyl diphosphate synthase, and (-)-pinene synthase. Arch. Biochem. Biophys. 392, 123-136.

Shishova, E.Y., Costanzo, L.D., Cane, D.E., Christianson, D.W., 2007. X-ray crystal structure of aristolochene synthase from Aspergillus terreus and evolution of templates for the cyclization of farnesyl diphosphate. Biochemistry 46, 1941-1951.

Srividya, N., Davis, E.M., Croteau, R.B., Lange, B.M., 2015. Functional analysis of (4S)-limonene synthase mutants reveals determinants of catalytic outcome in a model monoterpene synthase. Proc. Natl. Acad. Sci. USA $112,3332-3337$. 
Starks, C.M., Back, K., Chappell, J., Noel, J., 1997. Structural basis for cyclic terpene biosynthesis by tobacco 5-epi-aristolochene synthase. Science 277, 1815-1820.

Tholl, D., 2006. Terpene synthases and the regulation, diversity and biological roles of terpene metabolism. Curr. Opin. Plant. Biol. 9, 297-304.

Umehara, M., Hanada, A., Yoshida, S., Akiyama, K., Arite, T., Takeda-Kamiya, N., Magome, H., Kamiya, Y., Shirasu, K., Yoneyama, K., Kyozuka, J., Yamaguchi, S., 2008. Inhibition of shoot branching by new terpenoid plant hormones. Nature 455, 195-200.

Wilderman, P.R., Peters, R.J., 2007. A single residue switch converts abietadiene synthase into a pimaradienen specific cyclase. J. Am. Chem. Soc. $129,15736-15737$.

Williams, D.C., McGarvey, D.J., Katahira, E.J., Croteau, R., 1998. Truncation of limonene synthase preprotein provides a fully active 'pseudomature' form of this monoterpene cyclase and reveals the function of the amino-terminal arginine pair. Biochemistry 37, 12213-12220.

Wise, M.L., Croteau, R., 1999. In: Cane, D.E. (Eds), Comprehensive natural products chemistry: isoprenoids including carotenoids and steroids. Elsevier Science, Oxford, UK, Vol 2, pp. 97-153.

Xu, M., Wilderman, P.R., Peters, R.J., 2007. Following evolution's lead to 
a single residue switch for diterpene synthase product outcome. Proc. Natl. Acad. Sci. USA 104, 7397-7401.

Yoshikuni, Y., Ferrin, T.E., Keasling, J.D., 2006. Designed divergent evolution of enzyme function. Nature 440, 1078-1082.

Zerbe, P., Chiang, A., Bohlmann, J., 2012. Mutational analysis of white spruce (Picea glauca) ent-kaurene synthase (PgKS) reveals common and distinct mechanisms of conifer diterpene synthases of general and specialized metabolism. Phytochemistry 74, 30-39.

Zhou, K. and Peters, R.J., 2011. Electrostatic effects on (di)terpene synthase product outcome. Chem. Commun. 47, 4074-4080. 

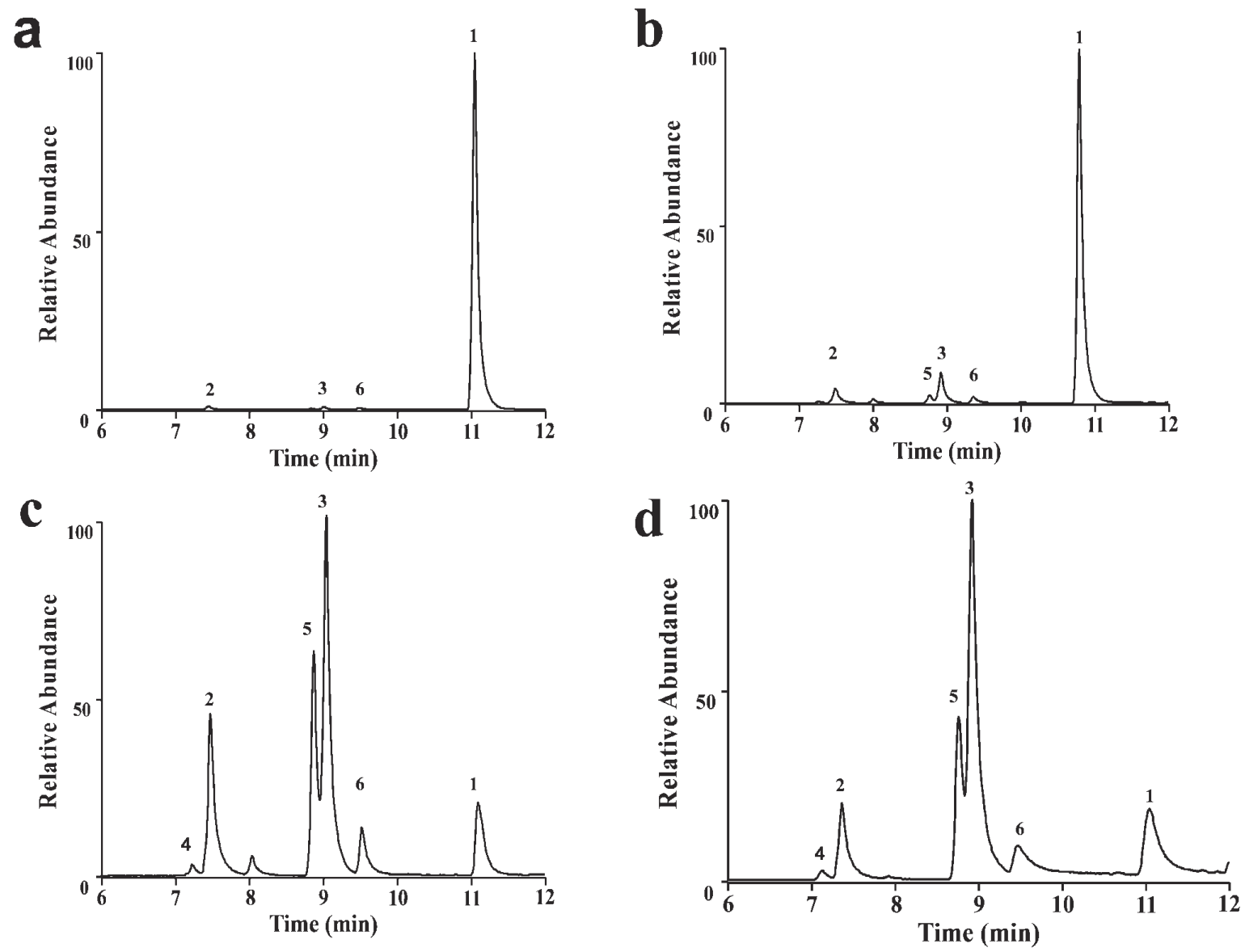


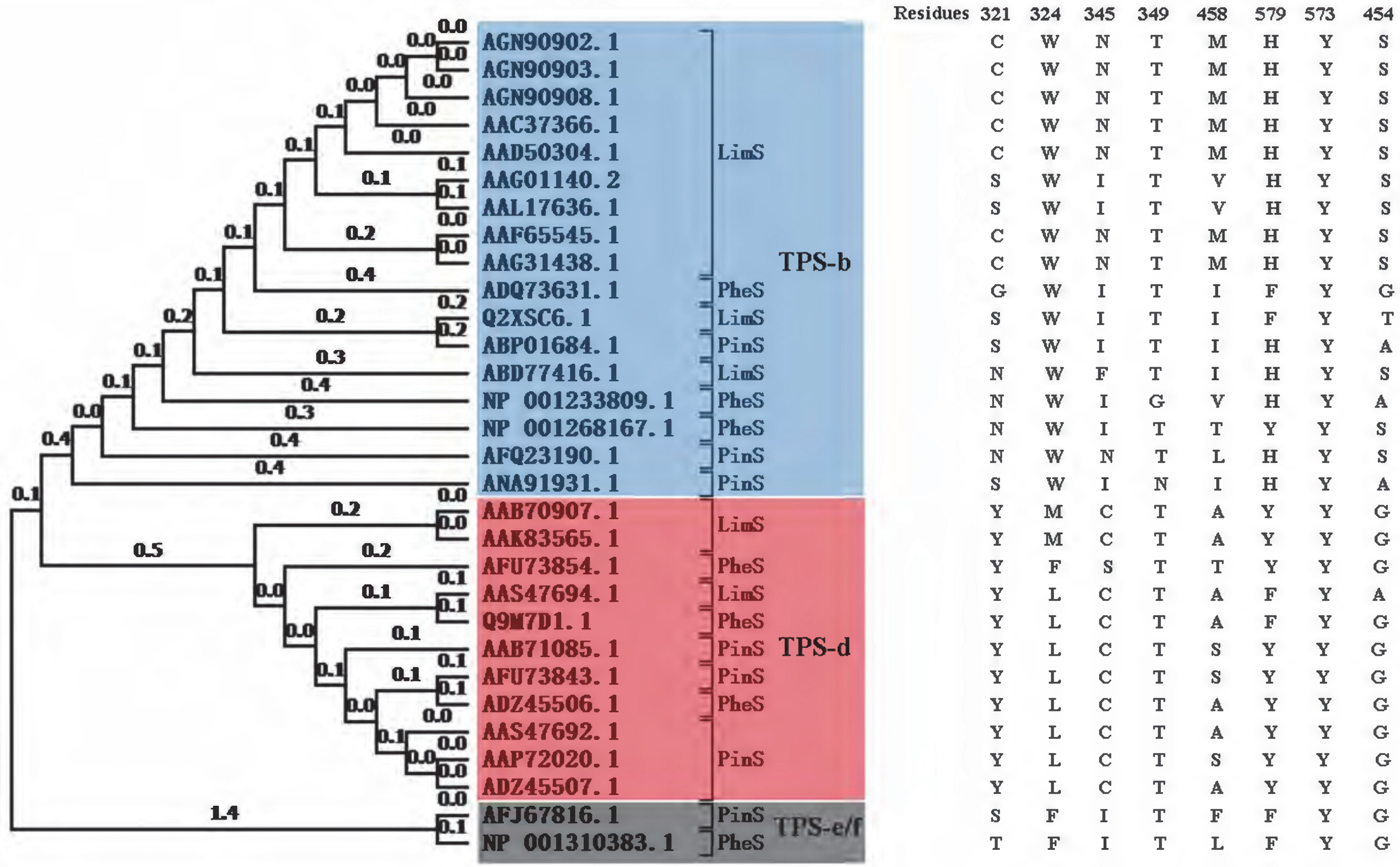

Residues $321 \quad 324 \quad 345 \quad 349$ 


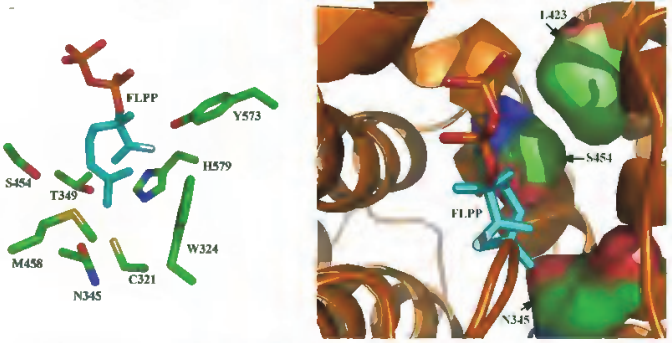

Mutation N345A/L423A/S454A converts $M$. spicata limonene synthase to a pinene synthase, while a single mutation N345 I converts it to a phellandrene synthase. Polar residues in the active pocket may be essential for limonene production by stabilizing the terpinyl cation. 